\title{
Coordination via Advection Dynamics in Nanonetworks with Molecular Communication
}

\author{
Malcolm Egan, Trang C. Mai, Trung Q. Duong and Marco Di Renzo
}

\begin{abstract}
A key challenge in nanonetworking is to develop a means of coordinating a large number of nanoscale devices. Molecular communication has emerged as a promising technique to assist in the coordination problem. Devices in molecular communication systems-once information molecules are releasedare typically viewed as passive, not reacting chemically with the information molecules. While this is an accurate model in diffusion-limited links, it is not the only scenario. In particular, the dynamics of molecular communication systems are more generally governed by reaction-diffusion, where the reaction dynamics can also dominate. This leads to the notion of reaction-limited molecular communication systems, where the concentration profiles of information molecules and other chemical species depends largely on reaction kinetics. In this regime, the system can be approximated by a chemical reaction network. In this paper, we exploit this observation to design new protocols for both point-topoint links with feedback and networks for event detection. In particular, using connections between consensus and advection theory and reaction networks lead to simple characterizations of equilibrium concentrations, which yield simple-but accuratedesign rules even for networks with a large number of devices.
\end{abstract}

\section{INTRODUCTION}

Nanonetworks consisting of nano-scale devices have been proposed to solve problems in environmental monitoring, health (in the form of nanomedicine), and a range of other domains [1]. A key challenge facing the design of nanonetworks is how these nano-scale devices can reliably exchange information. This is due to the limited resources of each device and their restricted ability to act in isolation. As such, collaboration is required for efficient sensing and actuation [2].

An emerging means of supporting communication between nano-scale devices is molecular communication. In molecular communication, information is encoded in the type, quantity or timing of molecules released by a transmitter. These molecules are then either carried, drift, or diffuse from the transmitter to a receiver, where decoding can take place.

Molecular communication systems where information molecules diffuse from the transmitter to the receiver has proven to be a popular design approach [3]. This is due to the

Malcolm Egan is with the Laboratoire CITI (a joint laboratory between the Université de Lyon, INRIA, and INSA de Lyon), 6 Avenue des Arts, 69621, Villeurbanne, France (malcom.egan@inria.fr). Trang C. Mai and Trung Q. Duong are with the School of Electronics, Electrical Engineering and Computer Science, Queen's University Belfast, UK ( $\{$ trung.q.duong,tmai01\}@qub.ac.uk). Marco Di Renzo is with the Laboratoire des Signaux et Systèmes, CNRS, CentraleSupélec, Univ Paris Sud, Université Paris-Saclay, 3 rue Joliot Curie, Plateau du Moulon, 91192, Gif-sur-Yvette, France. (e-mail: marco.direnzo@12s.centralesupelec.fr). His work is supported in part by the Agence Nationale de la Recherche Scientifique (ANR) through the research project SpatialModulation (Société de l'Information et de la Communication - Action Plan 2015). fact that free diffusion requires no external energy to transport information molecules from the transmitter to the receiver. In this approach, the main factor affecting the reliability of the transmission is randomness in the arrival time of each information molecule, leading to a noisy channel.

However, design based only on the fluid channel does not capture a number of important features of nano-scale devices. In particular, this approach neglects the chemical processes that produce information molecules at the transmitter and allow the receiver to decode information. For example, these reactions can create complex concentration profiles for the information molecules themselves as well as for the substrates used to produce and decode the information molecules. In this case, the standard impulse response obtained based only on diffusion [4] is not accurate. In particular, the channel response must be obtained by solving the reaction-diffusion equations that govern the system [5], which are typically difficult to solve analytically (for an important exception, see [6] for the study of a special case related to $\mathrm{Ca}^{2+}$ signaling).

Nevertheless, when the ratio between the characteristic time for diffusion and the characteristic time for the reactions to occur-known as the Damköhler number $D_{a}$, which we discuss later in more detail-is large compared with one, the pure diffusion models are accurate approximations of the reaction-diffusion equations. On the other hand, it is also possible to consider systems where the Damköhler number $D_{a} \ll 1$, where the effect of the chemical reactions in the transmitter and receiver dominate the diffusion. In this case, the reaction-diffusion equations are well approximated by chemical reaction networks [7], governed by systems of ordinary differential equations (ODEs) that determine the time evolution of the concentration for each type of molecule. As a consequence, the dynamics of the molecular communication link are constrained by the rates of the chemical reactions rather than the diffusion time of the information molecules.

In this paper, we explore the notion of reaction-limited molecular communication, where the dynamics of the communication link is dominated by the chemical reactions in the system rather than the effects of diffusion. This communication strategy can be contrasted with the standard approach based on diffusion, which can be identified as diffusion-limited molecular communication. A key advantage of the reactionlimited approach is a natural coupling or feedback between the transmitter and receiver, in the common situation where reactions are reversible. This feedback is exploited in nature by bacteria colonies in the form of quorum sensing [8], which provides a means of establishing a distributed consensus on 
the size of the colony.

In order to design reaction-limited molecular communication links, it is necessary to characterize the time-varying concentrations of each type of chemical species in the system. As these concentrations are determined by typically nonlinear systems of ODEs, closed-form characterizations are not feasible. Nevertheless, it is possible to obtain a simple characterization for the equilibrium concentrations of each chemical species. This is achieved by exploiting recent results that link chemical reaction networks to advection dynamics which are dual, in a sense explained later, to the well-known consensus problem [9], [10].

Using this characterization of the equilibrium concentrations we propose reaction-limited point-to-point molecular communication links with feedback. We also apply the same principles to develop event detection strategies in the presence of imperfect nano-scale sensing devices. In particular, we provide a design strategy to ensure that the network satisfies a maximum miss-detecton probability constraint. In order to optimize the detection time, we numerically optimize the nanonetwork by solving the system of ODEs governing the concentrations of each species in the network.

\section{SySTEM MOdEL}

\section{A. Chemical Reaction Network Preliminaries}

Before introducing a general model for reaction-limited molecular communication systems, we review concepts from the theory of chemical reaction networks that we will use in the remainder of the paper. To illustrate these concepts, we begin with the ubiquitous class of enzyme-activated chemical reaction networks. These networks consist of four chemical species: the enzyme $E$; the reactant $S$; the complex $\overline{E S}$; and the product $P$. The set of chemical species in this example is then $\mathcal{S}_{E}=\{E, S, \overline{E S}, P\}$. In this system there are three reactions:

$$
\begin{gathered}
E+S \stackrel{k_{3}}{\rightarrow} \overline{E S} \\
\overline{E S} \stackrel{k_{2}}{\rightarrow} E+S \\
\overline{E S} \stackrel{k_{\text {cat }}}{\longrightarrow} E+P,
\end{gathered}
$$

where $k_{1}, k_{2}, k_{\text {cat }}$ are called reaction rate coefficients.

A convenient way of representing each of these reactions is as a map from $\mathbb{N}^{\mathcal{S}_{E}}$ to $\mathbb{N}^{\mathcal{S}_{E}}$. For example, the first reaction is written as $(1,1,0,0) \rightarrow(0,0,1,0)$. In this way, we can define a set of reactions $\mathcal{R}_{E}=\left\{\mathbf{y}_{i} \rightarrow \mathbf{y}_{i}^{\prime}, i=1,2,3\right\}$, where $\mathbf{y}_{i} \in \mathbb{N}^{\mathcal{S}_{E}}$ is the vector of reactants in reaction $i$ and $\mathbf{y}_{i}^{\prime} \in \mathbb{N}^{\mathcal{S}_{E}}$ is the vector of products.

The pair of chemical species and chemical reactions $\left(\mathcal{S}_{E}, \mathcal{R}_{E}\right)$ is called a chemical reaction network. In order to characterize this network, we also need to consider the dynamics. Let $[E](t),[S](t),[\overline{E S}](t),[P](t)$ denote the concentration of each chemical species at time $t$. Under the standard assumption of mass-action kinetics, the concentrations of each species in the enzyme-activated system are governed by the following system of ODEs

$$
\begin{aligned}
& \frac{\mathrm{d}[E](t)}{\mathrm{d} t}=-k_{1}[E](t)[S](t)+k_{2}[\overline{E S}](t)+k_{c a t}[\overline{E S}](t) \\
& \frac{\mathrm{d}[S](t)}{\mathrm{d} t}=-k_{1}[E](t)[S](t)+k_{2}[\overline{E S}](t) \\
& \frac{\mathrm{d}[\overline{E S}](t)}{\mathrm{d} t}=k_{1}[E](t)[S](t)-k_{2}[\overline{E S}](t)-k_{c a t}[\overline{E S}](t) \\
& \frac{\mathrm{d}[P](t)}{\mathrm{d} t}=k_{c a t}[\overline{E S}](t),
\end{aligned}
$$

with initial conditions $[E](0)=E_{0},[S](0)=S_{0},[\overline{E S}](0)=$ $\overline{E S}_{0}$, and $[P](0)=P_{0}$. The biochemical system can then be written as the tuple $\left(\mathcal{S}_{E}, \mathcal{R}_{E}, k_{E}\right)$, where $k_{E}:\{1,2,3\} \rightarrow$ $\left\{k_{1}, k_{2}, k_{\text {cat }}\right\}$.

We now consider general chemical reaction systems.

Definition 1. A chemical reaction system is the tuple $(\mathcal{S}, \mathcal{R}, k)$ consisting of a set of chemical species $\mathcal{S}$, a set of reactions $\mathcal{R}=\left\{\mathbf{y}_{i} \rightarrow \mathbf{y}_{i}^{\prime}, i=1,2, \ldots\right\}$, and the rate function $k$.

Let $\mathbf{x}(t) \in \mathbb{R}^{\mathcal{S}}$ be the vector consisting of concentrations of each chemical species at time $t$. Under mass-action kinetics, the dynamics of the chemical reaction system is then governed by

$$
\dot{\mathbf{x}}(t)=\sum_{\mathbf{y} \rightarrow \mathbf{y}^{\prime} \in \mathcal{R}} k_{\mathbf{y} \rightarrow \mathbf{y}^{\prime}} \mathbf{x}(t)^{\mathbf{y}}\left(\mathbf{y}^{\prime}-\mathbf{y}\right),
$$

where $\mathbf{x}(t)^{\mathbf{y}}=x_{1}(t)^{y_{1}} x_{2}(t)^{y_{2}} \cdots$.

In general, it is not possible to obtain simple closedform solutions for the concentration trajectories $\mathbf{x}(t)$, which poses challenges for communication system design within this framework. Despite this, equilibrium solutions corresponding to $\mathbf{x}(t)$ as $t \rightarrow \infty$ can have a simpler characterization, when they exist. This fact forms a useful basis to develop communication protocols as we show in Section IV-A and Section IV-B.

\section{B. Reaction-Limited Molecular Communication}

Consider a nanonetwork consisting of $N$ devices that lie in a fluid. Each device is a container with a partially permeable surface. In device $i$, there is a set of chemical species $\mathcal{S}_{i}$. Each set $\mathcal{S}_{i}$ contains two types of chemical species, those that can permeate the device surface $\mathcal{I}_{i} \subseteq \mathcal{S}_{i}$ and those that cannot $\mathcal{A}_{i}=\mathcal{S}_{i} \backslash \mathcal{I}_{i}$. As such, the species in $\mathcal{I}_{i}$ can be used to carry information about the concentration of species $\mathcal{A}_{i}$ in each device throughout the network. On the other hand, the species $\mathcal{A}_{i}, i=1,2, \ldots, N$ form information sources for each device.

In general, it is possible to define a wide class of chemical reactions. However, for the purposes of this paper it is desirable to instead focus on a simpler class of reaction. In particular, we assume that for device $i$, the following firstorder reactions hold.

$$
\begin{aligned}
& A_{i} \stackrel{k_{A_{,} i}}{\rightarrow} I \\
& I \stackrel{k_{I_{I} i}}{\rightarrow} A_{i},
\end{aligned}
$$


where $k_{A, i}, k_{I, i}$ are the reaction rate coefficients for the reactions between the $i$-th species and the information molecule. An example of this class of reactions are isomerization reactions, which have been used for diffusion-limited molecular communications in [11].

Under the assumption of mass-action kinetics, the concentrations of each species in the network is governed by the reaction-diffusion equations

$$
\begin{aligned}
& \frac{\partial\left[A_{i}\right]}{\partial t}=D_{i} \nabla^{2}\left[A_{i}\right]-k_{A, i}\left[A_{i}\right]+k_{I, i}[I] \\
& \frac{\partial[I]}{\partial t}=D_{I} \nabla^{2}[I]-\sum_{i=1}^{N} k_{I, i}[I]+\sum_{i=1}^{N} k_{A, i}\left[A_{i}\right] .
\end{aligned}
$$

In general, it is not possible to solve this system of partial differential equations in closed form, even under simple boundary conditions. However, these equations simplify in the reactionlimited regime (where the dynamics in (3) dominate) or in the diffusion-limited regime. These two regimes are distinguished by the Damköhler number $D_{a}$ [5], which is the ratio of the characteristic time scales for the diffusion and the reactions. That is, given a reaction with rate $k$ and diffusion over a length $L$ with viscocity $D$, the Damköhler number is given by [12]

$$
D_{a}=\frac{k L^{2}}{D}
$$

In particular, the system is reaction-limited if $D_{a} \ll 1$ and diffusion-limited if $D_{a} \gg 1$. As we will show in the following section, reaction-limited systems have several desirable properties for use in nanonetworking, which are obtained by using reaction network theory ${ }^{1}$.

Remark 1. We have focused on the case of molecular communication systems governed by first-order reactions as this forms the basis for the protocols we develop in Section IV-A and Section IV-B. However, the framework of reaction-limited molecular communication can be readily generalized to more complicated networks of chemical reactions.

\section{REACTIONS NETWORKS AND CONSENSUS}

In the previous section, we have established a general model for reaction-limited molecular communication. However, in a practical communication network, it is necessary to be able to optimize design parameters to ensure reliable communication. For general reaction networks, it is not possible to obtain simple closed-form solutions for the system of ODEs in (3). Nevertheless, it is tractable to obtain simple characterizations of the equilibrium concentrations for useful classes of reaction networks, which we develop in this section based on recent connections between reaction networks and multi-agent consensus problems.

Consider the graph representation of a chemical reaction network, where each substrate and product is associated with the vertex of a directed graph $\mathcal{G}$, with edges corresponding to

\footnotetext{
${ }^{1}$ We remark that similar tools have recently been developed in the reactiondiffusion setting for the first-order reactions in (4) in [13]. We discuss extensions to this setting in Section VI.
}

reactions. In particular, suppose there are $c$ complexes in the reaction network, then the directed graph $\mathcal{G}$ with $c$ vertices and $r$ edges is called a graph of complexes, which is defined by the $c \times r$ incidence matrix D. In the model considered in Section II, a complex consists of only one species and therefore each vertex corresponds to a single species.

In fact, our model has linear dynamics due to the fact that there is only a single substrate and a single product in each reaction. In particular, the dynamics in (3) can be expressed in the form

$$
\dot{\mathbf{x}}(t)=\mathbf{D K} \mathbf{x}(t),
$$

where $\mathbf{K}$ is the matrix with $i$-th column consisting of the reaction coefficients for reactions with species $i$ as the substrate (called the outgoing co-incidence matrix in [10]). To establish the connection between the dynamics and the graph representation, let $\mathbf{L}=-\mathbf{D K}$. It follows immediately from the mass-action dynamics in (4) that $\mathbf{1 L}=\mathbf{0}$; i.e., the matrix $\mathbf{L}$ is a graph Laplacian, which gives rise to advection dynamics [9], [10]. In particular, there is a known equilibrium concentration, depending on the structure of the graph Laplacian and on the initial concentration.

In the case that the reaction rates $k_{A, i}=k_{I, i}$, then the further property $\mathbf{L} \mathbf{1}=\mathbf{0}$ holds. In this case, the dynamics in (7) are equivalent to consensus dynamics. As a consequence, we have the following theorem [14].

Theorem 1. If the graph Laplacian corresponding to the system in (7) satisfies $\mathbf{L} \mathbf{1}=\mathbf{0}$, then the dynamics in (7) converge globally and exponentially to to consensus point $\overline{\mathrm{x}} \mathbf{1}$, where $\overline{\mathbf{x}}$ is the average concentration over all species.

Remark 2. The link to consensus only holds in general for single substrate single product reaction networks. When each complex contains more than one species, then the dynamics are non-linear.

In the case that $k_{A, i}=k_{I, i}$ with $i=1, \ldots, N$, by Theorem 1, the equilibrium concentration $\mathbf{x}_{e}=\lim _{t \rightarrow \infty} \mathbf{x}(t)$ is given by

$$
\mathbf{x}_{e}=\frac{1}{N} \sum_{j=1}^{N} x_{j}(0) \mathbf{1} .
$$

The simplicity of this equilibrium characterization suggests that it might play a useful role in the design of reaction-limited communication systems. We explore this idea in the following sections.

We remark that it is also possible to consider other choices of $k_{A, i}, k_{I, i}$ that do not require the graph $\mathcal{G}$ to be balanced (for more details see [9, Proposition 10]. For the purposes of exposition, we focus on the case $k_{A, i}=k_{I, i}$ in the remainder of the paper.

\section{ApPliCATIONS}

\section{A. Molecular Communication with Feedback}

Consider a reaction-limited molecular communication system consisting of a transmitting and a receiving device, 
where the transmitter seeks to reliably transmit data to the receiver. The transmitting device encodes information in the concentration of the species $A_{1}$, which is enclosed within the transmitter membrane. Also present in the transmitter is another species which forms the information molecule $I$. At the receiver, there is another species $A_{2}$, which is enclosed in the receiver membrane. Only the information molecule is free to diffuse from the transmitter to the receiver.

As the system is reaction-limited, the concentration dynamics are well approximated by the chemical reactions, given by

$$
\begin{aligned}
& A_{1} \rightarrow I \\
& I \rightarrow A_{1} \\
& A_{2} \rightarrow I \\
& I \rightarrow A_{2} .
\end{aligned}
$$

By Theorem 1, it follows that the concentration of each species in this system will converge exponentially to an equilibrium

$$
\left[A_{1}\right](t) \rightarrow \frac{1}{3}\left(\left[A_{1}\right](0)+\left[A_{2}\right](0)+[I](0)\right) .
$$

An important practical issue is whether the transmitter can obtain feedback that a transmission is successful, which might arise when there are interfering molecules of the species $A_{1}, A_{2}$ or $I$. To this end, suppose that the system is initially at equilibrium and there is mass conservation, which means that

$$
\left[A_{1}\right](0)=\left[A_{2}\right](0)=[I](0) .
$$

A transmission corresponds to a change in the concentration of $A_{1}$ inside in the transmitting device. Suppose that this change leads to a new initial concentration $\left[A_{1}\right]\left(0^{+}\right)=\left[A_{1}\right](0)+\Delta$. It then follows that as $t \rightarrow \infty$ the new equilibrium concentration satisfies

$$
\left[A_{1}\right](t)=\left[A_{2}\right](t)=I(t) \rightarrow \frac{\left[A_{1}\right](0)+\left[A_{2}\right](0)+[I](0)+\Delta}{3}
$$

As such, the transmitter can detect interference by comparing the equilibrium concentration of $\left[A_{1}\right]$ with (12). The transmission has been corrupted if

$$
\left|\left[A_{1}\right](t)-\frac{\left[A_{1}\right](0)+\left[A_{2}\right](0)+[I](0)+\Delta}{3}\right|>\tau,
$$

where $\tau$ depends on the decoding regions at the receiving device.

We remark that there may also be sources of noise introduced by the fact that the equilibrium in (12) is obtained for the reaction-limited regime. In particular, small diffusion effects may induce variations in the equilibrium concentrations. Nevertheless (12) provides a simple design rule for the threshold.

To illustrate the behavior of the point-to-point link with feedback, we plot the time evolution of the concentration for each species. In particular, Fig. 1 shows the evolution of the concentrations of $A_{1}, A_{2}$ and $I$ with $\left[A_{1}\right](0)=$
$2 M,\left[A_{2}\right](0)=1 M,[I](0)=2 M$ with reaction rate coefficients $k_{A, 1}=1 s^{-1}, k_{A, 2}=0.5 s^{-1}, k_{I, 1}=1 s^{-1}, k_{I, 2}=$ $0.5 s^{-1}$, which ensures that the Laplacian $\mathbf{L}$ corresponding to the reaction network is balanced. Observe that the concentrations reach an equilibrium with the same value, which is given by (10) confirming that Theorem 1 holds in this setting.

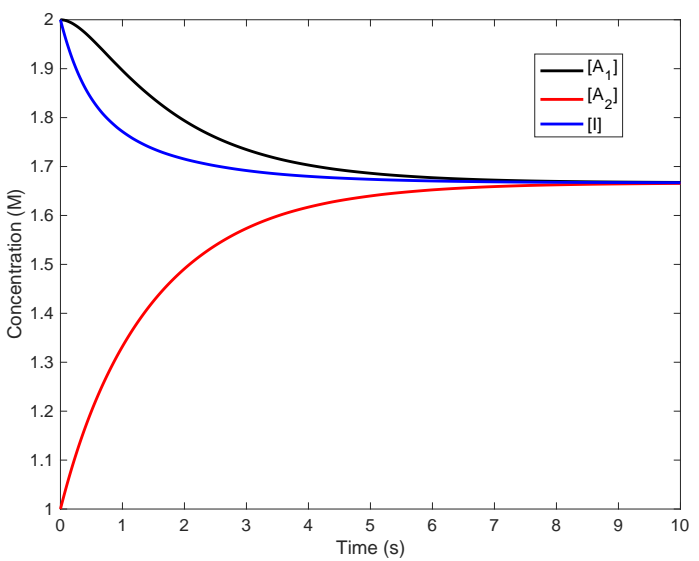

Fig. 1. Time evolution of the concentrations governed by (9) with $\left[A_{1}\right](0)=$ $2 M,\left[A_{2}\right](0)=1 M,[I](0)=2 M$ and $k_{A, 1}=1 s^{-1}, k_{A, 2}=$ $0.5 s^{-1}, k_{I, 1}=1 s^{-1}, k_{I, 2}=0.5 s^{-1}$.

In order to validate the need for the balanced Laplacian condition, we now change the reaction rate coefficient to be $k_{I, 2}=0.4 \mathrm{~s}^{-1}$ and plot the time evolution of the concentrations in Fig. 2. Observe that the concentrations still converge to an equilibrium, which follows from the general advection dynamics characterization of the equilibrium in [9, Proposition 10]. However, the concentrations at equilibrium do not agree as in the case of a balanced Laplacian.

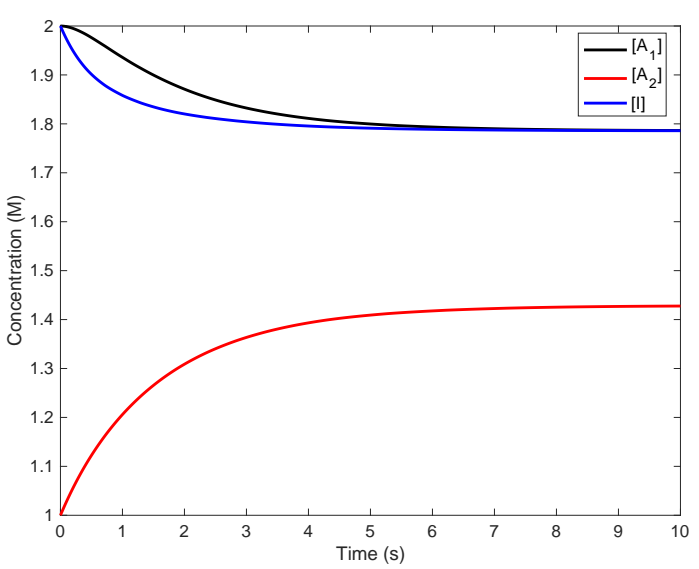

Fig. 2. Time evolution of the concentrations governed by (9) with $\left[A_{1}\right](0)=$ $2 M,\left[A_{2}\right](0)=1 M,[I](0)=2 M$ and $k_{A, 1}=1 s^{-1}, k_{A, 2}=$ $0.5 s^{-1}, k_{I, 1}=1 s^{-1}, k_{I, 2}=0.4 s^{-1}$.

Consider the case that the reaction rate coefficients are the same as in Fig. 1. Fig. 3 shows the effect of interference on the equilibrium concentrations. In particular, $\left[A_{1}\right](0)=$ 
$2 M,\left[A_{2}\right](0)=1 M,[I](0)=2.5 M$, where the initial concentration of $I$ is affected by interference. Observe that both the convergence to equilibrium and the final equilibrium concentrations are affected. For this reason, the presence of interference can be detected using the simple thresholding scheme in (13).

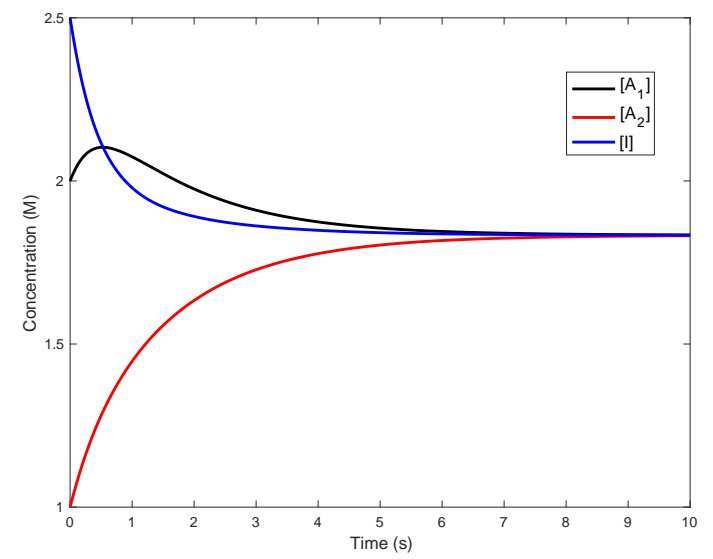

Fig. 3. Time evolution of the concentrations governed by (9) with $\left[A_{1}\right](0)=$ $2 M,\left[A_{2}\right](0)=1 M,[I](0)=2.5 M$ and $k_{A, 1}=1 \mathrm{~s}^{-1}, k_{A, 2}=$ $0.5 s^{-1}, k_{I, 1}=1 s^{-1}, k_{I, 2}=0.5 s^{-1}$.

\section{B. Nanonetworks for Event Detection}

In our first application, we focused on point-to-point communication links. We now turn to the problem of event detection, which is a nanonetwork coordination problem. In particular, consider a nanonetwork consisting of $N$ devices with receptors that are sensitive to the presence of malicious chemicals. In each period of time, the network seeks to converge to a consensus based on the presence or absence of these chemicals. We assume that each device is not completely reliable, with a miss-detection probability $p_{m i s s}$ and false alarm probability $p_{f a}$.

We suppose that the dynamics of the system follow (4) with $\left[A_{1}\right](0)=\cdots=\left[A_{K}\right](0)=\left[A_{I}\right](0)$. By Theorem 1, the equilibrium concentration of each species is given by

$$
\left[A_{i}\right]_{e}=\frac{\sum_{i=1}^{N}\left[A_{i}\right](0) X_{i}+[I](0)}{N+1},
$$

where $X_{i}$ is a Bernoulli random variable with success probability $1-p_{m i s s}$ in the case that an event occurs $\left(H_{1}\right)$ and $p_{f a}$ in the case that an event does not occur $\left(H_{0}\right)$. As such, the concentration $\left[A_{i}\right]_{e}$ is a scaled binomial random variable. This yields the likelihood ratio test [15]

$$
\Lambda=\left(\frac{1-p_{f a}}{p_{\text {miss }}}\right)^{N}\left[\frac{p_{\text {miss }} p_{f a}}{\left(1-p_{f a}\right)\left(1-p_{\text {miss }}\right)}\right]^{\frac{(N+1)\left[A_{i}\right] e}{\left[A_{i}\right](0)}} \underset{H_{1}}{\stackrel{H_{0}}{\gtrless}} \tau_{E}
$$

where $\tau_{E}$ is the threshold. The miss-detection probability corresponding to the hypothesis test is then given by $\operatorname{Pr}(\Lambda \leq$ $\left.\tau_{E} \mid H_{1}\right)$.
Observe that the detection protocol is dramatically simpler than in the case of diffusion-limited nanonetworks. This is due to the fact that hypothesis test in the case where the dynamics are dominated by diffusion depends on the hitting time distribution [16], [17], [18]. In general nanonetworks, the fluid may introduce anomalous diffusion where Brownian motion models are not accurate [19]. In this case, the hitting time distribution cannot be obtained in closed-form, which poses a challenge for deriving detection rules.

\section{The EfFect of Device Permeability}

In the model introduced in Section II, it was assumed that the membrane enclosing each device was perfectly permeable to the information molecule. We now explore how this model can be extended to include the effect of partial permeability, which is known to play an important role in the dynamics of reaction-diffusion systems such as in bacteria colonies [8].

To proceed, we assume that in each device $i$, the set of reactions includes

$$
\begin{aligned}
& A_{i} \stackrel{k_{A, i}}{\rightarrow} I_{i} \\
& I_{i} \stackrel{k_{I}, i}{\rightarrow} A_{i},
\end{aligned}
$$

which are the same as arising in Section II.

Once species $I_{i}$ passes through the membrane of a device, it diffuses through the fluid. Upon reaching another device, the species $I_{i}$ can also permeate through the membrane of the new device. To model this, we introduce the following reactions.

$$
\begin{aligned}
& I_{i} \stackrel{k_{I_{1}, f}}{\rightarrow} I_{f} \\
& I_{f} \stackrel{k_{f, I_{i}}}{\longrightarrow} I_{i},
\end{aligned}
$$

where $I_{i}$ is the information molecule in device $i$ and $I_{f}$ is the information molecule in the fluid. Note that each $I_{i}$ and $I_{f}$ are physically the same species. However, we have distinguished between the information molecules in the devices and in the fluid to capture the presence of imperfect permeability and that each device is an open reaction system. We remark that all the reactions in (16) and (17) are of first order and therefore the advection dynamics equilibrium characterization applies.

As in Section II, we assume mass-action dynamics, which means that the concentration of each species is governed by the following system of ODEs.

$$
\begin{aligned}
& \frac{\mathrm{d}\left[A_{i}\right]}{\mathrm{d} t}=-k_{A, i}\left[A_{i}\right]+k_{I, i}\left[I_{i}\right] \\
& \frac{\mathrm{d}\left[I_{i}\right]}{\mathrm{d} t}=-k_{I, i}\left[I_{i}\right]+k_{A, i}\left[A_{i}\right]+k_{f, I_{i}}\left[I_{f}\right]-k_{I_{i}, f}\left[I_{i}\right] \\
& \frac{\mathrm{d}\left[I_{f}\right]}{\mathrm{d} t}=\sum_{i=1}^{N} k_{I_{i}, f}\left[I_{i}\right]-k_{f, I_{i}}\left[I_{f}\right] .
\end{aligned}
$$

We remark that the rate coefficients $k_{I_{i}, f}$ and $k_{f, I_{i}}$ are determined by the permeability of the surface of the container.

Fig. 4 shows the effect of the permeable membrane. In this example, $\left[A_{1}\right](0)=2 M,\left[A_{2}\right](0)=1 M,\left[I_{f}\right](0)=$ $2 M,\left[I_{1}\right](0)=0.1 M,\left[I_{2}\right](0)=0.1 M$ with rate coefficients $k_{A, 1}=1 s^{-1}, k_{A, 2}=1 s^{-1}, k_{I_{1}}=1 s^{-1}, k_{I_{2}}=$ 


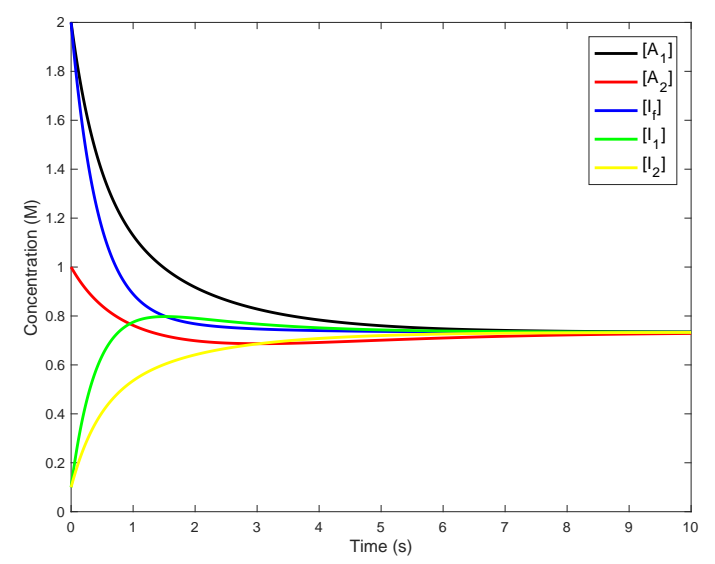

Fig. 4. Time evolution of the concentrations governed by (18 with $\left[A_{1}\right](0)=$ $2 M,\left[A_{2}\right](0)=1 M,\left[I_{f}\right](0)=2 M,\left[I_{1}\right](0)=0.1 M,\left[I_{2}\right](0)=0.1 M$ and $k_{A, 1}=1 \mathrm{~s}^{-1}, k_{A, 2}=0.5 \mathrm{~s}^{-1}, k_{I_{1}}=1 \mathrm{~s}^{-1}, k_{I_{2}}=0.5 \mathrm{~s}^{-1}, k_{I_{1}, f}=$ $1 s^{-1}, k_{I_{2}, f}=0.5 s^{-1}$.

$0.5 s^{-1}, k_{I_{1}, f}=1 s^{-1}, k_{I_{2}, f}=0.5 s^{-1}$, which ensure that the Laplacian is balanced. Observe that the concentrations converge to equilibrium as expected from Theorem 1. However, the effect of the membrane is to introduce a distinction between the information molecules inside each device and in the fluid. This leads to additional dynamics, which differ from Fig. 1 .

\section{CONCLUSIONS}

Existing proposals for molecular communication systems have largely focused on the dynamics governing the transport of molecules from transmitters to receivers. However, these dynamics only form part of the complete dynamics for the system, which is in fact governed by reaction-diffusion. As such, typical proposals fall in the diffusion-limited regime. In this paper, we have considered the dynamics introduced by reactions between information molecules and other chemical species in the system. Our approach therefore focuses on what can be called reaction-limited molecular communication.

In contrast with diffusion-limited dynamics, reaction-limited dynamics are governed by systems of ordinary differential equations. These differential equations are in general nonlinear, and as such it is challenging to obtain simple characterizations for the purpose of communication system design. However, in the special case of first-order chemical reactions (e.g., isomerization reactions), the dynamics are linear. A powerful tool in this setting is the link with advection on graphs, which provides simple characterizations of the equilibrium behavior, as time $t \rightarrow \infty$.

We have exploited the link with advection on graphs to design new protocols for feedback in point-to-point molecular communication systems, which is useful in identifying whether or not a transmission has been successful. We also showed that this approach is useful in event detection, facilitating threshold design in order to ensure that an event is detected, even when devices are not perfectly reliable.
There are a number of interesting further directions related to the protocols we have introduced, and also the reactionlimited molecular communication system design approach in general. For example, is it possible to introduce the effects of diffusion as noise in the observations of the concentrations in each device? A further avenue is to study molecular communication systems that are governed by the full reactiondiffusion dynamics. At present, it remains an open problem to obtain tractable characterization in this setting.

\section{REFERENCES}

[1] T. Nakano, M. Moore, F. Wei, A. Vasilaks, and J. Shuai, "Molecular communication and networking: opportunities and challenges," IEEE Trans. on Nanobioscience, vol. 11, pp. 135-148, Jun. 2012.

[2] I. Akyildiz, F. Brunetti, and C. Blázquez, "Nanonetworks: a new communication paradigm," Computer Networks, vol. 52, pp. 2260-2279, Aug. 2008.

[3] N. Farsad, H. B. Yilmaz, A. Eckford, C. B. Chae, and W. Guo, "A comprehensive survey of recent advancements in molecular communication," IEEE Commun. Surveys Tutorials, vol. 18, pp. 1887-1919, Third Quarter 2016.

[4] M. Pierobon and I. Akyildiz, "A physical end-to-end model for molecular communication in nanonetworks," IEEE Journal on Selected Areas in Communications, vol. 28, pp. 602-611, May 2010.

[5] H. Fogler, Elements of Chemical Reaction Engineering, 4th Ed. New York, NY: Prentice Hall, 2006.

[6] T. Nakano and T. Suda, "Molecular communication using dynamic properties of oscillating and propagating patterns in concentration of information molecules,' IEEE Transactions on Communications, vol. 65, pp. 3386-3398, Aug. 2017.

[7] M. Feinberg, "Chemical reaction network structure and the stability of complex isothermal reactors-i. the deficiency zero and deficiency one theorems," Chemical Engineering Science, vol. 42, no. 10, pp. 22292268, 1987.

[8] P. Melke, P. Sahlin, A. Levchenko, and H. Jönsson, "A cell-based model for quorum sensing in heterogeneous bacteria colonies," PLoS Computational Biology, vol. 6, Jun. 2010.

[9] A. Chapman and M. Mesbahi, "Advection on graphs," in IEEE Conference on Decision and Control and European Control Conference (CDCECC), (Orlando, FL), Dec. 2011.

[10] A. van der Schaft, S. Rao, and B. Jayawardhana, "A network dynamics approach to chemical reaction networks," International Journal of Control, vol. 89, no. 4, 2016.

[11] N.-R. Kim and C.-B. Chae, "Novel modulation techniques using isomers as messenger molecules for nano commuication networks via diffusion," IEEE Journal on Selected Areas in Communications, vol. 31, pp. 847856, Dec. 2013.

[12] R. Brodkey and H. Hershey, Transport Phenomena: A Unified Approach. New York, NY: McGraw-Hill, 1988.

[13] K. Fellner, W. Prager, and B. Tang, "The entropy method for reactiondiffusion systems without detailed balance: first order chemical reaction networks," Kinetic and Related Models, vol. 10, no. 4, pp. 1055-1087, 2017.

[14] L. Moreau, "Stability of continuous-time distributed consensus algorithms," in IEEE Conference on Decision and Control (CDC), (Nassau, Bahamas), Dec. 2004.

[15] H. Trees, Detection, Estimation and Modulation Theory, Part I. John Wiley and Sons, 2004.

[16] H.-J. Chiu, L. S. Meng, P. C. Yeh, and C. H. Lee, "Near-optimal low complexity receiver design for diffusion-based molecular communication," in IEEE Global Communications Conference (GLOBECOM), (Atlanta, GA), Dec. 2013.

[17] B. Li et al., "Local convexity inspired low-complexity noncoherent signal detector for nanoscale molecular communications," IEEE Trans. Commun., vol. 64, pp. 2079-2091, May 2016.

[18] Y. Fang, A. Noel, N. Yang, A. Eckford, and R. Kennedy, "Maximum likelihood detection for collaborative molecular communication," arXiv:1704.05623, 2017.

[19] T. Mai, M. Egan, T. Duong, and M. Di Renzo, "Event detection in molecular communication networks with anomalous diffusion," IEEE Communications Letters, vol. 21, pp. 1249-1252, Feb. 2017. 